\title{
Efficacy of chlorhexidine against catheter-related infections
}

Catheter-related infections commonly occur in the health-care setting and are associated with high mortality. Research led by Olivier Mimoz and the CLEAN trial investigators now shows that disinfecting a patient's skin with an alcohol formulation of $2 \%$ chlorhexidine-alcohol is more effective in decreasing the incidence of catheterrelated infections and catheter colonization than is $5 \%$ povidone iodine-alcohol. Furthermore, scrubbing the skin with detergent before disinfection did not reduce the incidence of catheter colonization.

\section{$\mathbf{4}$...these new findings will have important consequences in reducing morbidity... 77}

The open-label, randomized, controlled trial included 2,349 patients from 11 intensive care units across France who required at least one central venous, haemodialysis, or arterial catheter. The study end points were assessed by physicians blinded to the study groups.
A total of 1,181 patients were assigned to receive chlorhexidine-alcohol and 1,168 patients were assigned to receive povidone iodine-alcohol. Half the patients in each group also underwent skin scrubbing with a detergent before antiseptic application. Skin colonization was evaluated before catheter removal and blood samples were obtained in cases in which catheter-related infection was suspected.

The researchers confirmed that a formulation of $2 \%$ chlorhexidine in $70 \%$ isopropyl alcohol was the most effective first-line antiseptic solution for skin preparation before catheter insertion and during catheter care. This formulation was associated with a sixfold lower incidence of catheter-related infections and a fivefold decrease in catheter-related bloodstream infections compared to povidone iodinealcohol. Scrubbing the skin with a detergent before antiseptic application had no significant effect on catheter colonization in either group $(P=0.3877)$.

"Previous single centre studies (including one from my centre) had shown that chlorhexidine in alcohol should probably be the preferred antiseptic formulation, but the study end points were not strong enough to make a definitive conclusion", explains Mimoz. "This large, multicentre study was able to reach sufficient power to demonstrate a significant impact of chlorhexidine on catheter-related infection".

The researchers believe that these new findings will have important consequences in reducing morbidity, mortality, and costs associated with catheter-related infections. They now plan to conduct a similar study in the operating room context to look for similar results in preventing surgical site infections.

Jessica K. Edwards 